\title{
Aplicación del shot peening en implantes dentales de titanio para la mejora de la osteointegración
}

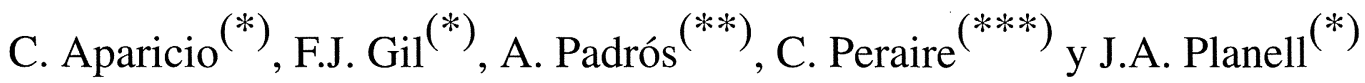

\begin{abstract}
Resumen Con el propósito de optimizar la fijación entre el implante y el hueso, se han realizado tratamientos de shot peening con distintos tipos de partículas de proyección: $\mathrm{TiO}_{2}, \mathrm{Al}_{2} \mathrm{O}_{3}$ y $\mathrm{SiC}$. La rugosidad superficial obtenida será colonizada por hueso neoformado que producirá un anclaje implante-hueso. Para valorar la influencia que cada tipo de material abrasivo tiene en la colonización del hueso sobre las superficies tratadas, se han realizado ensayos in vitro de cultivos osteoblásticos. El shot peening se ha llevado a cabo en discos de titanio comercialmente puro, sobre los que se ha caracterizado cuantitativa y cualitativamente la rugosidad después del tratamiento; así como la contaminación de la superficie por las partículas. También se ha determinado el tamaño de las partículas, antes y después del tratamiento, para valorar sus porcentajes de rotura. Finalmente, se ha propuesto un proceso de fabricación de partículas de $\mathrm{TiO}_{2}$ por sinterización en el laboratorio. La fabricación ha sido necesaria debido a la imposibilidad de encontrar en el mercado este tipo de partículas.
\end{abstract}

Palabras clave: Shot peening. Titanio. Osteointegración. Implante dental. Rugosidad superficial.

\section{Osseointegration improvement by shot peening in titanium dental implants}

\begin{abstract}
In order to optimize the implant-bone fixation, different shot peening treatments with different shot particles $\left(\mathrm{TiO}_{2}, \mathrm{Al}_{2} \mathrm{O}_{3}, \mathrm{SiC}\right)$ have been made. The influence that each type of shot particle has in the bone colonization on the different treatment surfaces has been determined by means of osteoblastlike cells culture. Commercially pure titanium discs have been shot peened. Their qualitative and quantitative surface roughness have been characterized; as well as their surface contamination caused by the shot particles. Particle size has also been determined, before and after the treatment, in order to evaluate their breaking averages. Finally, a $\mathrm{TiO}_{2}$ shot particles manufacture process by sintering has been developed. The manufacture has been necessary since this type of shot particles are not available in the market with the adequate size.
\end{abstract}

Keywords: Shot peening. Titanium. Osseointegration. Dental implant. Surface roughness.

\section{INTRODUCCIÓN}

La rugosidad superficial es una variable que influye de forma determinante en el buen comportamiento del implante dental de titanio comercialmente puro (Ti c.p.), tanto a corto como a largo plazo. En este sentido, se han realizado estudios in

(*) Dpto. de Ciencia de los Materiales e Ingeniería Metalúrgica. E.T.S.I.I. Univ. Politécnica de Cataluña. Diagonal 647; 08028-Barcelona (España).

(**) Clínica Pad, Barcelona (España)

(***) C.I.D.A.S.A.L. Centro de Investigación y Desarrollo Aplicado S.A.L. Santa Perpètua de Mogoda. Barcelona (España). vivo que demuestran que una cierta rugosidad superficial mejora la fijación del implante al hueso (1-3) Por otra parte, estudios in vitro han puesto de manifiesto que la diferenciación y proliferación osteoblástica, así como la producción de matriz ósea, también resultan influidas positivamente por el aumento de la rugosidad de la superficie (4-6).

Un procedimiento ya estudiado y utilizado para incrementar la rugosidad en la superficie de los implantes dentales es el shot peening. Este procedimiento, que consiste en proyectar partículas de elevada dureza a gran velocidad y presión sobre la superficie del implante, es adecuado porque, además de dar rugosidad superficial, elimina posibles 
defectos del mecanizado de la pieza; limpia la superficie de contaminantes; aumenta la vida a fatiga del implante, debido al aumento de la tensión compresiva en la superficie, y aumenta su resistencia a la corrosión bajo tensión (7).

Una de las variables más importantes del shot peening es la naturaleza de las partículas utilizadas para ser proyectadas contra la superficie del metal a tratar. En primer lugar, esta importancia viene determinada por el hecho de que, después del tratamiento superficial, limpieza, pasivado y esterilización del implante, siempre quedan restos de partículas procedentes del shot peening que, con posterioridad a la implantación, pueden desprenderse y pasar a los tejidos circundantes. Para evitar esta contaminación, se puede, por ejemplo, proyectar partículas de la misma composición química que la superficie del implante, es decir, $\mathrm{TiO}_{2}$. También se pueden buscar otras naturalezas que influyan menos negativamente o que presenten la posibilidad de eliminarse de la superficie del implante con más facilidad.

Por otro lado, la naturaleza de la partícula es también relevante por la capacidad que cada una tiene para realizar el trabajo mecánico necesario para conseguir la rugosidad deseada.

Además, desde un punto de vista de costes de fabricación, que las partículas utilizadas se rompan más o menos, tiene una gran influencia, hecho que también es importante desde un punto de vista técnico, ya que, si se rompen menos, va a mejorar la homogeneidad en la rugosidad de la superficie.

Por estos motivos, se hace necesario investigar la influencia que el tipo de partículas va a tener sobre la respuesta de los implantes dentales de Ti c.p.

\section{MATERIALES Y MÉTODOS}

\subsection{Preparación de los discos}

Los discos objeto de estudio tienen $6 \mathrm{~mm}$ de diámetro y son de Ti c.p. Se les ha realizado un tratamiento superficial de shot peening, con una presión de chorro de 0,25 MPa, durante $5 \mathrm{~s}$.

Se han preparado distintos grupos de discos en función del tipo de partícula de proyección: $\mathrm{Al}_{2} \mathrm{O}_{3}$, $\mathrm{TiO}_{2}$ y $\mathrm{SiC}$. En todos los casos, se han utilizado partículas de gran tamaño $(425-600 \mu \mathrm{m})$. Todos los discos se han pasivado con $\mathrm{HCl}$ y esterilizado con óxido de metileno.

\subsection{Sinterización de óxido de titanio}

Se han sinterizado, en un horno de mufla, un total de $1.800 \mathrm{~g}$ de polvo de $\mathrm{TiO}_{2}$ (fase rutilo) químicamente puro, con la siguiente operativa:
- Se llenan 9 crisoles de alúmina con $200 \mathrm{~g}$ de polvo de $\mathrm{TiO}_{2}$ cada uno.

- Se comprime el polvo dentro del crisol con ayuda de una maza.

- Dos a dos, los crisoles se introducen en el horno a $600{ }^{\circ} \mathrm{C}$.

- Se sube la temperatura hasta $1.000{ }^{\circ} \mathrm{C}$, y se mantiene durante $13 \mathrm{~h}$.

- Transcurrido dicho tiempo, se extraen los crisoles y se dejan enfriar al aire desde $1.000{ }^{\circ} \mathrm{C}$.

De cada crisol se ha obtenido un bloque sinterizado de óxido de titanio. Para obtener las partículas definitivas, cada bloque se ha disgregado hasta tamaños reducidos de aproximadamente $30 \mathrm{~g}$ y estos se han triturado con maza y mortero. El producto triturado se ha cribado con tamices normalizados hasta conseguir partículas del tamaño deseado.

\subsection{Tamaño de partícula}

El ensayo de determinación del tamaño de partícula se ha realizado para una muestra de cada uno de los tipos de partícula $\left(\mathrm{Al}_{2} \mathrm{O}_{3}, \mathrm{TiO}_{2}\right.$ y SiC); antes (sin usar) y después (usado) del tratamiento de shot peening.

La determinación del tamaño de partícula se ha llevado a cabo por el método de difracción de rayos láser.

\subsection{Rugosidad}

Los ensayos de rugosidad superficial sobre los discos tratados se han realizado con un rugosímetro para medidas bidimensionales, con palpador de diamante de forma cónica $\left(90^{\circ}\right)$ con $5 \mu \mathrm{m}$ de diámetro en la punta, de una resolución mínima de $0,1 \mu \mathrm{m}$. Los valores numéricos de los diferentes parámetros de rugosidad superficial, se calcularon con un software adecuado adaptado al rugosímetro.

Se han analizado 5 discos de cada uno de los tipos implantados, realizándose 3 lecturas de rugosidad para cada uno. Para cada una de las lecturas, se ha tomado una longitud de muestra de $0,8 \mathrm{~mm}$, analizándose 3 muestras, es decir, la longitud total de ensayo para cada lectura ha sido de $2,4 \mathrm{~mm}(0,8$ $\mathrm{mm} \times 3$ ). Además, se ha ensayado con un pretravel -avance del palpador previo a la determinación del perfil, que tiene por objeto estabilizar la posición del disco antes de iniciar la lectura- de $0,4 \mathrm{~mm}$.

Se ha utilizado un filtro Gaussiano con longitud de corte igual a la longitud de muestra, $0,8 \mathrm{~mm}$. Así, partiendo del perfil que detecta el rugosímetro, se filtran las longitudes de onda superiores a la de corte, obteniendo como resultado el perfil de rugo$\operatorname{sidad} R$, a partir del cual se calculan los parámetros. 
Los parámetros obtenidos en cada lectura han sido:

$\boldsymbol{R} \boldsymbol{a}$ (Media aritmética de las desviaciones del perfil). Es la media aritmética del valor absoluto de las distancias desde la línea media al perfil $R$ dentro de la longitud de muestra. Esta línea media es una línea de referencia para el cálculo de los distintos parámetros, que se determina por el método de los mínimos cuadrados respecto al perfil $R$ en cada longitud de muestra. $R a$ se calcula en micrómetros y es el parámetro más general y comúnmente utilizado para describir la rugosidad.

$\boldsymbol{R} \boldsymbol{y}$ (Máxima altura del perfil). Es un valor extremo, y describe la distancia entre la altura del máximo pico y la profundidad del mayor valle, dentro de la longitud de muestra.

$\boldsymbol{R z}$ (Altura de diez puntos irregulares). Es el valor medio, en micrómetros, del valor absoluto de los cinco picos más altos, más el valor medio del valor absoluto de los cinco valles más profundos, dentro de la longitud de muestra. Este parámetro es sensible a los cambios pronunciados en las características topográficas.

Las imágenes de la superficie de los discos, para realizar análisis cualitativos sobre el aspecto rugoso de las muestras, se han obtenido con ayuda de un microscopio electrónico de barrido.

\subsection{Composición superficial}

Para analizar la composición superficial y, por lo tanto, la posible contaminación de la superficie de los discos por las partículas del shot peening, se ha utilizado un analizador de dispersión de energías de rayos X, "Analytical LZ-5 (Link, R.U.)". Se ha analizado la cantidad porcentual atómica de los elementos más significativos para cada muestra, con ensayos de $100 \mathrm{~s}$ y $20 \mathrm{kV}$ de energía del haz de rayos $\mathrm{X}$.

\subsection{Estudios osteoblásticos}

Se distribuyeron los discos de titanio en diferentes placas estériles y se sembraron con una suspensión celular de 12.100 células $/ \mathrm{cm}^{2}$ y se incubaron a $37{ }^{\circ} \mathrm{C}$ en una atmósfera húmeda, que contenía 5,5 $\%$ de $\mathrm{CO}_{2}$. Como control negativo se utilizaron discos de poliestireno.

Al cabo de $24 \mathrm{~h}$, a la mitad de las muestras (Grupo A) se les cambió el medio de cultivo por $200 \mu \mathrm{L}$ de medio IMDM + 3\% de suero delipidado con carbono activo con vitamina $\mathrm{K}_{1}$ y vitamina $\mathrm{C}$. El resto de muestras (Grupo B) recibieron el mismo medio, pero con vitamina $\mathrm{D}$, lo que produce la inducción de osteocalcina. Tras $72 \mathrm{~h}$ de incubación se recolectó el medio de cultivo de cada una de las muestras en tubos Eppendorf. Se tripsinizaron los cultivos y se realizó un recuento celular; para ello, se determinaron 5 campos en cada muestra y se obtuvo el valor medio. Se determinaron los niveles de osteocalcina para cada una de ellas.

\section{RESULTADOS Y DISCUSIÓN}

\subsection{Sinterización de óxido de titanio}

El proceso de fabricación de partículas por sinterización de polvo de $\mathrm{TiO}_{2}$, ofreció el siguiente resultado: de cada $1.800 \mathrm{~g}$ de polvo introducidos en el horno, se obtuvieron aproximadamente $200 \mathrm{~g}$ de partículas con el tamaño deseado. Esto significa un rendimiento en peso muy pobre, de sólo un $11 \%$.

A este bajo rendimiento, se añade lo laborioso del proceso y, sobre todo, las pobres características mecánicas que han demostrado estas partículas, como ponen de manifiesto los ensayos de rotura de partícula y de rugosidad superficial que más adelante se analizan.

\subsection{Rotura de partícula}

En la tabla I se muestran los porcentajes de rotura de partícula, calculados a partir de las medidas de diámetro medio antes y después de ser usadas en el proceso de shot peening.

A la vista de los resultados se aprecia que las partículas que más se rompen son las de óxido de titanio, más del doble que las de carburo de silicio y casi siete veces más que las de alúmina. Esto refuerza los comentarios anteriores y aporta un dato significativo: las partículas de carburo de silicio son más frágiles que las de óxido de aluminio; hecho importante desde el punto de vista de la optimización del proceso de shot peening.

TABLA I.- $\%$ de rotura de los tres tipos de partículas utilizados para realizar el shot peening, sobre discos de Ti c.p.

TABLE I.- \% of breaking of the three types of particles used on c.p. Ti peened discs

\begin{tabular}{|c|c|}
\hline Tipo de partícula & $\begin{array}{l}\% \text { de rotura } \\
\left(\left(1-\frac{\text { usado }}{\sin \text { usar }}\right) \cdot 100\right.\end{array}$ \\
\hline $\begin{array}{c}\mathrm{Al}_{2} \mathrm{O}_{3} \\
\mathrm{SiC} \\
\mathrm{TiO}_{2}\end{array}$ & $\begin{array}{r}3,67 \\
12,25 \\
25,06\end{array}$ \\
\hline
\end{tabular}




\subsection{Rugosidad}

$R a$ es el parámetro que se emplea habitualmente para valorar el efecto de la rugosidad de la superficie de los implantes en la respuesta biológica y funcional de los mismos; por este motivo, los análisis de comparación entre los distintos tratamientos se describen y se caracterizan con él. El resto de los parámetros no han aportado diferencias en las conclusiones obtenidas con $R a$.

Los valores de la tabla II demuestran que la rugosidad superficial obtenida en los discos realizando el shot peening con $\mathrm{TiO}_{2}(R a=0,54 \mu \mathrm{m})$ es claramente inferior a la obtenida trabajando con $\mathrm{Al}_{2} \mathrm{O}_{3}(R a=4,42 \mu \mathrm{m})$ y con SiC-HCl $(R a=5,00$ $\mu \mathrm{m})$. Este resultado es contradictorio con otros trabajos en los que se ha utilizado el $\mathrm{TiO}_{2}$ como material de proyección en el shot peening (8-10). Es de reseñar que, en estos trabajos, los tamaños de partícula utilizados han sido mucho más pequeños que los del presente estudio. Es evidente, pues, que el método de fabricación, por sinterización, de partículas de $\mathrm{TiO}_{2}$ que se ha llevado a cabo en el presente estudio, no es adecuado para su utilización como material de proyección en el shot peening.

Por otra parte, se observa que la diferencia entre la rugosidad superficial conseguida con $\mathrm{Al}_{2} \mathrm{O}_{3}$ y $\mathrm{SiC}$ es significativa ( $\mathrm{p}$-valor $=0,0058$ ), como se observa en la tabla III, aunque sólo ligeramente: $\Delta R a=0,6 \mu \mathrm{m}$. Este hecho puede deberse a una

TABLA II: Caracterización de la rugosidad superficial de discos de Ti c.p. en función de la partícula de proyección utilizada en el tratamiento de shot peening. (D.E. = Desviación Estándar). $R a$ : media aritmética de las desviaciones del perfil. $R z$ : altura de diez puntos irregulares. $R q$ : media de la raíz cuadrada de las desviaciones del perfil

TABLE II: Surface roughness characterization of c.p. Ti discs as a function of the shot particles used in shot peening treatment. (D.E. = Standard Deviation). Ra: arithmetical mean deviation of the profile. $\mathrm{Rz}$ : mean-square-root deviation of the profile. $\mathrm{Rq}$ : ten point height of irregularities

\begin{tabular}{|r|c|c|r|r|}
\hline & $\begin{array}{c}\text { Sin shot } \\
\text { peening }\end{array}$ & $\mathrm{TiO}_{2}$ & $\mathrm{Al}_{2} \mathrm{O}_{3}$ & $\mathrm{SiC}$ \\
\hline $\mathrm{Ra}(\mu \mathrm{m})$ & 0,331 & 0,540 & 4,423 & 4,995 \\
media & 0,112 & 0,109 & 0,356 & 0,631 \\
$\mathrm{DE}$ & & & & \\
\hline $\mathrm{Rz}(\mu \mathrm{m})$ & 1,709 & 2,961 & 17,191 & 18,532 \\
media & 0,587 & 0,617 & 0,678 & 2,116 \\
$\mathrm{DE}$ & & & & \\
\hline $\begin{array}{r}\mathrm{Ry}(\mu \mathrm{m}) \\
\text { media }\end{array}$ & 3,063 & 4,713 & 26,167 & 29,406 \\
$\mathrm{DE}$ & 1,047 & 1,539 & 3,278 & 3,559 \\
\hline
\end{tabular}

TABLA III: p-valores de las comparaciones de las medias de rugosidad superficial $(R a)$, en discos de Ti c.p., obtenidas con distintos tratamientos de shot peening en función del tipo de partícula de proyección utilizado. Los p-valores se han obtenido realizando ensayos de comparación de medias de t-Student. La diferencia entre las medias es estadísticamente significativa si el p-valor es igual o inferior a 0,05

TABLE III: p-values of the surface roughness mean comparisons (Ra), on c.p. Ti discs, obtained with different shot peening treatments as a function of the shot particle type used. The p-values have been obtained by $t$-Student tests. Difference between the means are statistically significant when p-value is equal or less than 0,05

\begin{tabular}{|l|c|c|}
\hline $\begin{array}{l}\text { Comparación } \\
\text { de medias }\end{array}$ & p-valor & Significatividad \\
\hline $\mathrm{SiC}$ vs $\mathrm{Al}_{2} \mathrm{O}_{3}$ & 0,0058 & SÍ \\
$\mathrm{Al}_{2} \mathrm{O}_{3}{\text { vs } \mathrm{TiO}_{2}}$ & 0,0000 & SÍ \\
$\mathrm{SiC}_{\mathrm{vs} \mathrm{TiO}_{2}}$ & 0,0000 & SÍ \\
$\mathrm{TiO}_{2}$ vs Sin tratamiento & 0,0000 & SÍ \\
\hline
\end{tabular}

mayor dureza de las partículas de $\mathrm{SiC}$, conclusión que se vería reforzada por su mayor fragilidad, puesta de manifiesto en los ensayos de rotura de partícula. Sin embargo, también cabe señalar que puede deberse a diferencias en la forma de las partículas, variable no controlada en el presente estudio.

Las imágenes, obtenidas con microscopio electrónico de barrido, de la superficie de los discos tratados con $\mathrm{TiO}_{2}$ (Fig. 1); con $\mathrm{Al}_{2} \mathrm{O}_{3}$ (Fig. 2); y con

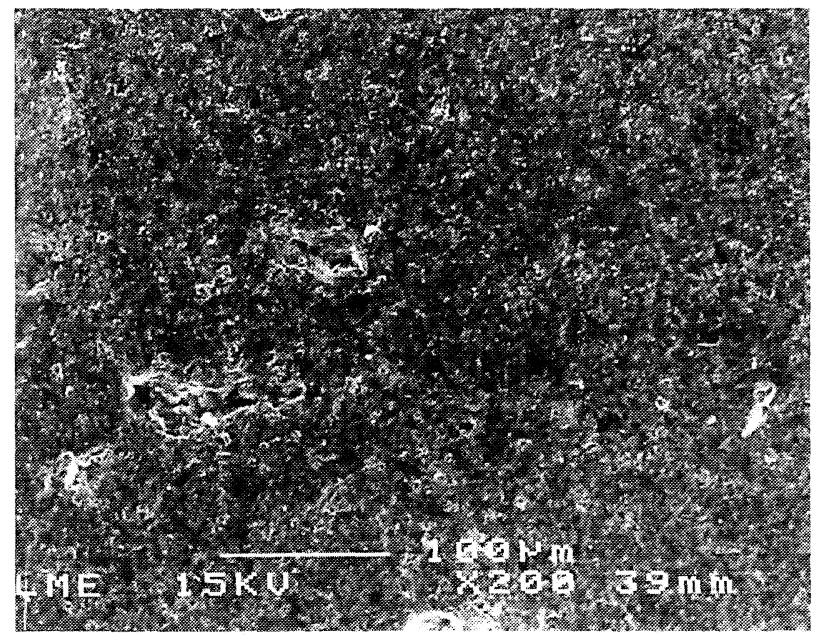

Fig. 1.- Micrografía de la superficie de un disco de Ti c.p. tratado con shot peening con partículas de proyección de $\mathrm{TiO}_{2}(\times 200)$.

FIG. 1.- Micrograph of the surface of a c.p. Ti disc treated by shot peening with $\mathrm{TiO}_{2}$ shot particles $(\times 200)$. 


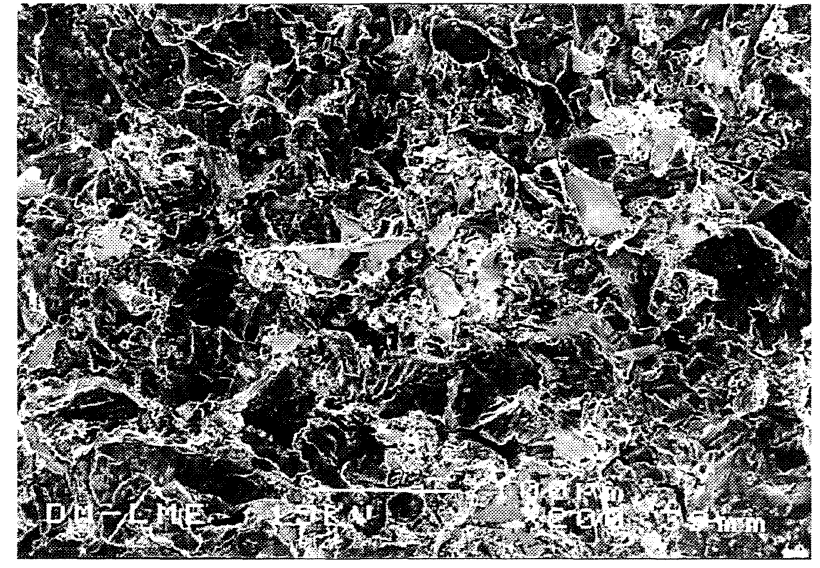

FIg. 2.- Micrografía de la superficie de un disco de Ti c.p. tratado con shot peening con partículas de proyección de $\mathrm{Al}_{2} \mathrm{O}_{3}(\times 200)$.

FIG. 2.- Micrograph of the surface of a c.p. Ti disc treated by shot peening with $\mathrm{Al}_{2} \mathrm{O}_{3}$ shot particles $(\times 200)$.

SiC (Fig. 3) refuerzan todos estos resultados. También, en las micrografías, se ven con claridad las partículas procedentes del shot peening que han quedado incrustadas en la superficie de los discos.

\subsection{Composición superficial}

Los resultados obtenidos con el microanálisis de dispersión de energías de rayos $\mathrm{X}$ han demostrado que el tratamiento con $\mathrm{TiO}_{2}$ no ha contaminado la superficie del implante con elementos ajenos, como era de esperar. Desde este punto de vista, sigue siendo importante valorar la posibilidad de conse-

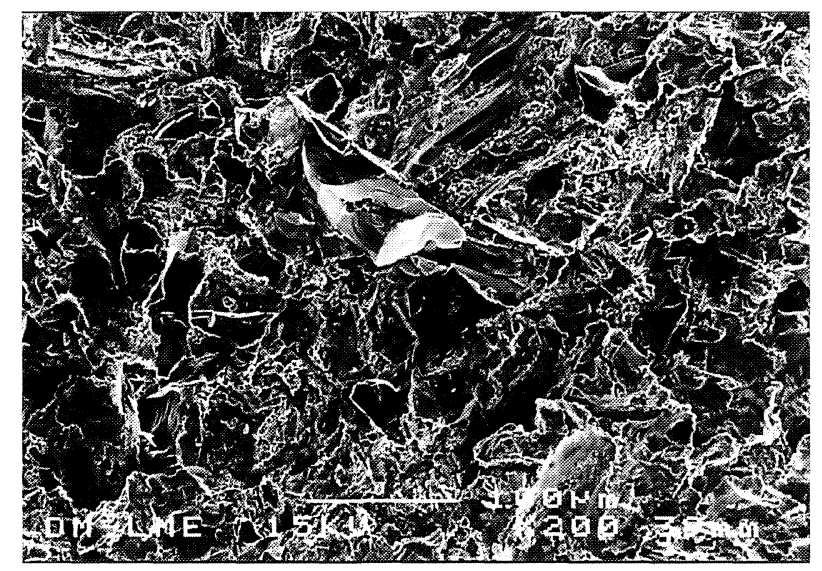

Fig. 3.- Micrografía de la superficie de un disco de Ti c.p. tratado con shot peening con partículas de proyección de $\mathrm{SiC}(\times 200)$.

FIG. 3.- Micrograph of the surface of a c.p. Ti disc treated by shot peening with $\mathrm{SiC}$ shot particles $(\times 200)$. guir el óxido por otras vías para poder realizar el tratamiento del shot peening de forma mecánicamente eficaz.

Por otra parte, no ha habido diferencias significativas entre los porcentajes atómicos de aluminio (22 $\%$ para el tratamiento con $\mathrm{Al}_{2} \mathrm{O}_{3}$ ) y silicio (30\% para el tratamiento con $\mathrm{SiC}$ ) encontrados en la superficie de los discos. Por lo que el siguiente paso es valorar la influencia que estos restos van a tener en el crecimiento de las células óseas sobre el Ti c.p.

\subsection{Crecimiento osteoblástico}

Ninguno de los materiales presentó signos de citotoxicidad, siendo todos los porcentajes inferiores al $25 \%$. En la tabla IV se muestran los recuentos de células osteoblásticas -precursoras de huesoen cada una de las superficies. Se puede apreciar que las superficies tratadas con $\mathrm{Al}_{2} \mathrm{O}_{3}$ son sobre las que proliferan mayor cantidad de células osteoblásticas, a pesar de que tienen menor rugosidad que las tratadas con SiC. Por tanto, en principio, serán sobre las que más rápida fijación implante-hueso cabrá esperar, debido a un posible efecto de estimulación de la reparación del tejido óseo por parte de las partículas de $\mathrm{Al}_{2} \mathrm{O}_{3}$ que quedan adheridas en la superficie.

Además, el grupo de las tratadas con $\mathrm{Al}_{2} \mathrm{O}_{3}$, permite una óptima adherencia celular, estadísticamente significativa respecto al resto de tratamientos (pvalor $<0,05$, test de Newman-Keuls). Los

TABLA IV: Recuento celular, expresado en número de cél $/ 0,01 \mathrm{~cm}^{2}$, para cada una de las superficies de titanio tratadas con diferentes partículas de proyección. El valor para $\mathrm{Al}_{2} \mathrm{O}_{3}$ es estadísticamente significativo con respecto al resto de tratamientos para ambos grupos (p-valor $<0,05$, ensayo NewmanKeuls). GRUPO A: cultivo con vitaminas $\mathrm{K}_{1}$ y $\mathrm{C}$.

GRUPO B: cultivo con vitaminas $K_{1}, C$ y D

TABLE IV: Cell count, in $10,01 \mathrm{~cm}^{2}$, on each titanium treated surfaces with different shot particles. The value of $\mathrm{Al}_{2} \mathrm{O}_{3}$ is statistically significant in relation to the rest of treatments in both groupes (p-value<0,05, Newman-Keuls test). GROUP A: culture with vitamines $K_{1}$ and C. GROUP B: culture with vitamines $K_{l}, C$ and $D$

\begin{tabular}{|l|c|c|}
\hline $\begin{array}{l}\text { Naturaleza } \\
\text { de las partículas }\end{array}$ & GRUPO A & GRUPO B \\
\hline $\mathrm{Al}_{2} \mathrm{O}_{3}$ & 52,80 & 50,07 \\
$\mathrm{SiC}$ & 29,67 & 27,40 \\
$\mathrm{TiO}_{2}$ & 20,93 & 28,20 \\
Sin tratamiento & 21,00 & 17,33 \\
\hline
\end{tabular}


osteoblastos presentan una morfología típica de superficies reactivas, lo cual indica junto a los elevados índices de osteocalcina que las células están activas y dispuestas a pasar a estados más diferenciados.

\section{CONCLUSIONES}

El proceso de fabricación de partículas de $\mathrm{TiO}_{2}$ por sinterización que se ha propuesto no es adecuado, ya que las partículas obtenidas carecen de las propiedades mecánicas necesarias para su utilización en el shot peening.

Con la utilización de SiC como partícula de proyección en el proceso de shot peening sobre Ti c.p., se obtiene una rugosidad superficial mayor -aprox. $\Delta R a=0,6-$ que si se utiliza como partícula de proyección el $\mathrm{Al}_{2} \mathrm{O}_{3}$, ambos tipos con tamaños grandes de partícula $(425-600 \mu \mathrm{m})$.

Desde el punto de vista de los costes del proceso, la utilización de las partículas de $\mathrm{Al}_{2} \mathrm{O}_{3}$ es la óptima de las tres utilizadas en este estudio, ya que poseen un porcentaje de rotura inferior.

Los estudios osteoblásticos otorgan a las superficeis tratadas con $\mathrm{Al}_{2} \mathrm{O}_{3}$ el mayor crecimiento osteoblástico en la superficie del metal, lo que en principio se traducirá en una mayor y más rápida fijación entre el implante y el hueso.

\section{Agradecimiento}

Los autores desean agradecer a la empresa Klockner S.A. y a D. Guillermo Nussbaum y D.
Agustín García, de la empresa Materias Primas Abrasivas S.L., la donación del material y equipos con los que se ha llevado a cabo la presente investigación, así como sus comentarios tan valiosos para el desarrollo de este trabajo.

\section{REFERENCIAS}

(1) Predecki, P., Stephan, J.E., Auslaender, B.A., MoOney, V.L. y Kirkland, K. J. Biomed. Mater. Res. 6, 1972: 375-400.

(2) Buser, D., Schenk, R.K., Steinemann, S., Fiorelini, J.P., Fox, C.H. y STICH, H. J. Biomed Mater. Res. 25, 1991: 889-902

(3) Johansson, C.B., Wenneberg, A., Han, C-H. y Albrektsson, T. Proc. 5th World Biomaterials Cong. Canadian Biomaterials Soc. Toronto (Canadá) 1996: 478.

(4) Bowers, K.T., Keller, J.C. y Michaels, C.M. Int. J. Oral Maxillofac Implants 7, 1992: 302.

(5) Groessner-Schreiber, B. y Tuan, R.S. J. Cell Sci., 101, 1992: 209

(6) Martin, J.Y., Schwartz, Z., Hummert, T.W., Schraub, D.M., Simpson, J., LANKFord, Jr J., DEAN, D.D., Cochran, D.L. y Boyan, B.D. J. Biomed. Res. 29, 1995: 389-401.

(7) Oshida, Y., Sachdeva, R., Miyazaki, S. y Daly, J. J. Mater. Sci. Mater. Med. 4, 1993: 443-447.

(8) Wenneberg, A., Albrektsson, T. y Andersson, B. $J$. Mater. Sci. Mater. Med. 6, 1995: 302-309.

(9) Wenneberg, A. y Albrektsson, T. Proc. 5th World Biomaterials Cong. Canadian Biomaterials Soc. Toronto (Canadá) 1996: 459.

(10) Wenneberg, A., Albrektsson, T., Johansson, C. y ANDERSSON, B. Biomaterials, 17, 1996: 15-22. 